\title{
Health and urban poverty
}

Next June, Recife will host the 1st Brazilian Workshop on Health in Subnormal Urban Agglomerates, the new term used by the Brazilian Institute of Geography and Statistics (IBGE) to refer to the socio-economically disadvantaged territories and populations situated on the peripheries of large- and medium-sized cities, previously known as favelas, slums or shanty-towns in the sociological literature in Latin America.

This pioneering event will occur (and this is the main reason for it) in an historical, political, demographic and epidemiological context that is highly characteristic and very important. Last year (2012), for the first time in human history, the urban population of the planet outstripped the rural, concluding the process of migration from the countryside to the city that became a characteristic of the advent of the Industrial Revolution and speeded up significantly in the second half of the past century.

Sociologists, anthropologists and economists have closely followed this process, which, although driven primarily by internal migration, is complemented, to a significant extent, by the vegetative growth of the urban population itself. In clear contrast to the approaches adopted by the social sciences, health sciences have accorded less importance to the implications of the process of urbanization on health and disease.

There can be no doubt that the 2012 United Nations mobilization, by way of the Fund for the Protection of Children and Adolescents, represented, above all, by the United Nations Children's Fund (UNICEF), is producing a new global interest in slum-dwellers. In a broader more international context, the United Nations has already agreed, in its so-called Millennium Goals (2000), to direct international efforts towards reducing the misery that is especially heavily concentrated in urban settlements, and which is directly connected with the poverty that holds sway in the rural parts of less developed countries. This means, by way of commitment to joint action, tackling the problem of unemployment and underemployment, of ethically unacceptable occupations, such as prostitution or drugs dealing, as well as controlling various forms of violence, dealing with the two main facets of sanitation (drinking water and sanitary sewerage), and addressing the issues of cramped and insalubrious dwellings, the lack of basic public services and, even, in extreme situations, the absence of the State as a political and institutional representative of society.

These are situations in which organized crime often takes on the functions of the State, as a virtual parallel government, with armed (and often rival) groups under the command of factions that maintain a climate of constant violence. This is, in fact, an ecosystem that is different in terms of its origins, structures and roles, organized and functioning as a political and social ghetto within the urban spaces created by social exclusion. This is a situation that is of concern not only to Brazil but to the whole world, with its disparities, intersecting interests, and shared challenges.

What kind of health conditions would one expect to find in such adverse social and environmental circumstances? Clearly not good ones. How is the epidemiological transition, which has changed patterns of morbidity and mortality from one generation to the next, playing itself out among low-income populations?

Unfortunately, there are no solid and reliable data on these developments. And it is certainly high time answers were sought, in a country with inequalities as striking as those of Brazil, which is one of the six largest economies in the world, but ranks in the bottom tier in terms of the quality of life of its population. We thus outstrip most rich countries in terms of Gross Domestic Product (GDP), while we rank lower than most poorer or developing countries in terms of the social dividend that is produced by the economic elite but does not benefit all. The health situation is set in this context of contrasts and restrictions.

Thus, the First Workshop on Health in the Subnormal Urban Agglomerates of Brazil will be held in a highly appropriate setting, both internationally, and, above all, in terms of our own internal problems and chal- 
lenges. The event to be held in Recife provides a unique opportunity to pool the experiences of the various regions of the country: the North, which has the largest population of slumdwellers; the Northeast, where Recife, Salvador and Fortaleza are the urban centers most affected by the formation of slums; the Southeast, where the ghettoes of Rio de Janeiro and São Paulo loom large in terms of international renown for urban criminality and, finally, the Mid-West and South, where poor communities, marginalized and stigmatized by the violence of organized criminal groups, in various cities in the State of Santa Catarina have begun to gain notoriety in the national and international news media.

Two days of debates will almost certainly not be enough provide a qualitative and quantitative overview of the range of health problems that lurk in the still little known world of Brazilian slums. Much still needs to be found out about the epidemiological profile of these populations, the performance of the public health services and the social security network, and government policy and action and its effectiveness.

However, it is hoped that the programed event will achieve consensus with regard to the little that is already known, the great deal that needs to be done and, as a result, the enormous amount that needs to be accomplished in order to obtain more and better information, through multi-professional and interdisciplinary studies, of the health situation of slum populations and the most obvious determining factors. This is the setting that is the focus of the National Workshop on the Health of Slum Populations in Brazil, which the promoters expect to be attended by representatives of the public health authorities and international United Nations agencies.

\section{Malaquias Batista Filho 1}

${ }^{1}$ Instituto de Medicina Integral Prof. Fernando Figueira. Rua dos Coelhos, 300. Boa Vista. Recife, PE, Brasil. CEP: 50.070550. E-mail: mbatista@imip.org.br 